\title{
Efficiency of equilibria in uniform matroid congestion games
}

\author{
Jasper de Jong ${ }^{1}$, Max Klimm² , and Marc Uetz ${ }^{1}$ \\ 1 Universiteit Twente, Enschede, The Netherlands \\ $\{j \cdot$ dejong-3,m.uetz\}@utwente.nl \\ 2 Technische Universität Berlin, Berlin, Germany \\ klimm@math.tu-berlin.de
}

\begin{abstract}
Network routing games, and more generally congestion games play a central role in algorithmic game theory, comparable to the role of the traveling salesman problem in combinatorial optimization. It is known that the price of anarchy is independent of the network topology for non-atomic congestion games. In other words, it is independent of the structure of the strategy spaces of the players, and for affine cost functions it equals $4 / 3$. In this paper, we show that the dependence of the price of anarchy on the network topology is considerably more intricate for atomic congestion games. More specifically, we consider congestion games with affine cost functions where the strategy spaces of players are symmetric and equal to the set of bases of a $k$-uniform matroid. In this setting, we show that the price of anarchy is strictly larger than the price of anarchy for singleton strategy spaces where the latter is $4 / 3$. As our main result we show that the price of anarchy can be bounded from above by $28 / 13 \approx 2.15$. This constitutes a substantial improvement over the price of anarchy bound $5 / 2$, which is known to be tight for arbitrary network routing games with affine cost functions.
\end{abstract}

1998 ACM Subject Classification C.2.1 Network Architecture and Design: Network topology F.2.2 Nonnumerical Algorithms and Problems: Computations on Discrete Structures I.2.1 Applications and Expert Systems: Games

Keywords and phrases Atomic congestion games, Price of anarchy, Matroid strategy spaces, Affine cost functions

\section{Introduction}

Understanding the impact of selfish behavior on the performance of a system is an important question in algorithmic game theory. One of the cornerstones of the substantial literature on this topic is the famous result of Roughgarden and Tardos [26]. They considered the traffic model of Wardrop [29] in a network with affine flow-dependent congestion cost functions on the edges. Given a set of commodities, each specified by a source node, a target node, and a flow demand, a Wardrop equilibrium is a multicommodity flow with the property that every commodity uses only paths that minimize the cost. For this setting, Roughgarden and Tardos proved that the total cost of an equilibrium flow is not worse than $4 / 3$ times that of a system optimum. This ratio was coined the price of anarchy by Koutsoupias and Papadimitriou [18] who introduced it as a measure of a system's performance degradation due to selfish behavior. A surprising consequence of the result of Roughgarden and Tardos is that the worst case price of anarchy in congested networks is attained for very simple single-commodity networks already considered a century ago by Pigou [22]. Pigou-style networks consist of only two nodes connected by two parallel links. In fact, Roughgarden [25] proved that for any set of cost functions, the price of anarchy is independent of the network topology as it is always 
attained for such simple Pigou-style networks where a feasible strategy of each commodity is to choose exactly one out of the two links.

A model closely related to Wardrop's model is that of a congestion game with unsplittable (i.e., atomic) players. In such a game, there is a finite set of players and a strategy of each player is to choose a set of resources allowable to her. Without any restrictions on the strategy spaces, the price of anarchy for affine cost functions is $5 / 2$ as shown by Christodoulou and Koutsoupias [9] and Awerbuch et al. [5]. As a contrast, for simple Pigou-style instances with symmetric and singleton strategies, Lücking et al. [19] showed that the price of anarchy is only $4 / 3$. These results imply that for atomic congestion games the price of anarchy does depend on the combinatorial structure of the players' strategies.

In this work, we shed new light on the impact of the combinatorial structure of strategy spaces on the inefficiency of equilibria in atomic congestion games. Specifically, we focus on the minimum combinatorial structure that one may think of, namely symmetric $k$-uniform congestion games where the strategy set of each player consists of all subsets of resources with exactly $k$ elements. These games are a natural generalization of the singleton case and model, e.g., load balancing scenarios where each player controls several jobs, see also Abed et al. [1] for a related model in the context of coordination mechanisms.

\subsection{Our Results}

As our main result we prove that the price of anarchy in congestion games with affine cost functions is at most 28/13 when strategy spaces are symmetric and bases of a $k$-uniform matroid. The proof uses in its core several combinatorial arguments on the amount and cost of resources that are over- respectively under-demanded in any given Nash equilibrium as opposed to an optimal solution. It also exploits the affinity of the cost functions, along the lines of earlier arguments of Fotakis [11] for the singleton case. The main point of the technical side of the paper is the insight that the combinatorial structure of strategy spaces, here of the simplest possible form, allows to furnish combinatorial arguments that yield improved results on the price of anarchy. We are not aware of earlier attempts in this direction, and believe this opens new possibilities for our understanding of a "classical" showcase problem in algorithmic game theory. We also show that the price of anarchy for the $k$-uniform matroid case cannot be the same as for singleton congestion games, as we bound it away from 4/3: For $k$ large enough, the price of anarchy is at least 1.343 for affine cost functions, for $k=5$ it is at least $47 / 35 \approx 1.3428$. We note that larger lower bounds on the price of anarchy can be achieved for more general settings such as matroid strategy spaces, or non-affine cost functions. They are not included in this paper, however.

\subsection{Related Work}

Since the early works of Pigou [22], Beckman et al. [6], and Braess [7] it is well known that user equilibria in congested networks may be suboptimal for the overall performance of the system. In order to quantify this inefficiency, Koutsoupias and Papadimitriou [18] proposed to study the ratio of the total cost of an equilibrium and the total cost of an optimal solution. This ratio is now known as the price of anarchy. Roughgarden and Tardos [26] showed that the price of anarchy for non-atomic games with affine costs is $4 / 3$. The worst case is attained for simple networks of two parallel links previously studied by Pigou [22]. Roughgarden [25] gave a closed form expression for the price of anarchy for arbitary cost functions which is again attained for Pigou-style networks, e.g., for polynomials with positive coefficient and maximum degree $d$ the price of anarchy is of order $\Theta(d / \ln (d))$. 
Awerbuch et al. [5] and Christodoulou and Koutsoupias [9] considered the related model with atomic players that was introduced by Rosenthal [23]. They showed that for affine cost functions the price of anarchy is 5/2. Aland et al. [4] gave tight bounds on the price of anarchy for polynomial cost functions with maximum degree $d$ which behaves asymptotically as $\Theta\left((d / \ln d)^{d+1}\right)$. It is interesting to note that these worst-case bounds are not attained for simple Pigou-style networks with symmetric and singleton strategies as in the non-atomic case. Based on previous work of Suri et al. [27], Caragiannis et al. [8] showed that for affine costs, the worst case is attained for asymmetric singleton strategies. For a similar result for polynomial costs, see Gairing and Schoppmann [14]. In fact, for singleton games with symmetric strategies, the price of anarchy is considerably better than in the general case. In fact, Fotakis [11] showed that the price of anarchy of symmetric singleton atomic games is equal to the price of anarchy of non-atomic games. This improves and generalizes previous bounds by Lücking et al. [19] and Gairing et al. [13].

The class of $k$-uniform games that we consider in this paper is also related to the class of integer-splittable congestion games introduced by Rosenthal [24] and the classes of $k$-splittable and integer $k$-splittable congestion games studied by Meyers [20]. In contrast to our model, the models above allowed that a player uses a resource with multiple units of demand at the same time. It turns out that allowing for this kind of self-congestion has a severe impact on the existence of pure Nash equilibria [10, 24] but for networks of parallel links it is known that pure Nash equilibria are guranteed to exist [16, 28].

The impact of combinatorial structure on the existence and computability of pure Nash equilibria in has been studied for many variants of congestion games. Ackermann et al. [2] proved that for atomic games with unweighted players all sequences of best replies converge in polynomial time to a pure Nash equilibrium if the set of strategies of each player corresponds to the set of bases of a matroid. For weighted congestion games, the matroid property guarantees the existence of a pure Nash equilibria [3] while without that property a pure Nash equilibrium may fail to exist [15]. Similarly, congestion games with player-specific costs and matroid strategies have a pure Nash equilibrium which can be computed efficiently [3] which is in contrast to the general case [21]. For similar results in the context of resource buying games, see also Harks and Peis [17].

To the best of our knowledge, the impact of matroid structures on the efficiency of Nash equilibria has not been considered before. The only result in this direction is a yet unpublished work of Fujishige et al. [12]. They showed that Braess' paradox cannot occur in non-atomic games with matroid strategies, i.e., the quality of the user equilibrium cannot deteriorate when removing a resource. This result, however, has no consequences for the inefficiency of equilbria in non-atmomic games since the worst case is attained for Pigou-style networks where the strategies are symmetric and 2-uniform matroids.

\section{Preliminaries}

Let $N=\{1, \ldots, n\}$ be a finite set of players and let $R$ be a finite set of resources. Each player $i$ is associated with a set of subsets of resources $X_{i} \subset 2^{R}$ allowable to her. A strategy of a player is to choose a subset $x_{i} \in X_{i}$ from this set. A strategy vector $x=\left(x_{i}\right)_{i \in N}$ consists of $n$ strategies, one for each player. Every resource $r$ is endowed with a cost function $c_{r}: \mathbb{N} \rightarrow \mathbb{R}$ that maps the total number of its users $x_{r}=\left|\left\{i \in N: r \in x_{i}\right\}\right|$ to a cost value $c_{r}\left(x_{r}\right)$. The private cost of player $i$ in strategy vector $x$ is then defined as

$$
\pi_{i}(x)=\sum_{r \in x_{i}} c_{r}\left(x_{r}\right) .
$$


We use standard game theory notation; for a strategy vector $x \in X=X_{1} \times \cdots \times X_{n}$, a player $i$ and an alternative strategy $y_{i} \in X_{i}$, we denote by $\left(y_{i}, x_{-i}\right)$ the strategy vector in which all players play as in $x$ except for $i$ who plays $y_{i}$. A strategy vector $x$ is a Nash equilibrium if,

$$
\pi_{i}(x) \leq \pi_{i}\left(y_{i}, x_{-i}\right) \text { for all } i \in N \text { and } y_{i} \in X_{i} .
$$

Given an instance of a game $I=\left(N, R, X,\left(c_{r}\right)_{r \in R}\right)$, we denote the set of Nash equilibria of $I$ by $\operatorname{NE}(I)$.

We are interested in how restrictions on the set of strategies of each player influence the inefficiency of equilibria. We measure the efficiency of a strategy vector $x \in X$ in terms of the social costs $C(x)$ defined as

$$
C(x)=\sum_{i \in N} \pi_{i}(x) .
$$

We denote by $\mathrm{OPT}(I)$ the set of strategy vectors $x$ that minimize $C(x)$. For an instance $I$ of a game, the price of anarachy is defined as

$$
\operatorname{PoA}(I)=\max _{x^{\mathrm{NE}} \in \mathrm{NE}(I)} \frac{C\left(x^{\mathrm{NE}}\right)}{C\left(x^{\mathrm{OPT}}\right)},
$$

where $x^{\mathrm{OPT}} \in \mathrm{OPT}(I)$ is a strategy vector minimizing $C$. For a class $\mathcal{G}$ of games, the price of anarachy is defined as

$$
\operatorname{PoA}(\mathcal{G})=\sup _{I \in \mathcal{G}} \operatorname{PoA}(I)
$$

We drop $\mathcal{G}$ whenever it is clear from context. We are specifically interested in singleton and uniform matroid strategy spaces. A game is said to be a singleton game, if $\left|x_{i}\right|=1$ for all $x_{i} \in X_{i}$ and $i \in N$. A game is called $k$-uniform game if for each player, there is a subset $R_{i} \subseteq R$ such that

$$
X_{i}=\left\{S \subseteq R_{i}:|S|=k\right\} .
$$

A game is called symmetric, if $X_{i}=X_{j}$ for all $i, j \in N$.

\section{Symmetric $k$-Uniform Games}

The main result of this paper is the following upper bound on the price of anarchy of symmetric $k$-uniform matroid congestion games.

- Theorem 1. The price of anarchy of symmetric k-uniform matroid congestion games is at most $\frac{28}{13} \approx 2.15$.

For the proof of Theorem 1 , we are going to to prove that $C\left(x^{\mathrm{NE}}\right) \leq \frac{28}{13} C\left(x^{\mathrm{OPT}}\right)$ for any given worst-case Nash equilibrium $x^{\mathrm{NE}}$ and optimal solution $x^{\mathrm{OPT}}$, of an abritrary instance $I$ of a $k$-uniform matroid congestion game. For the remainder of this section, fix an instance $I$, a worst-case Nash equilibrium $x^{\mathrm{NE}}$ and a system optimal solution $x^{\mathrm{OPT}}$.

To gain some intuition on congestion games with $k$-uniform matroid strategies, let us first consider the following example of a $k$-uniform matroid congestion game that will serve as a running example throughout this section. Even though it has only a moderate price of anarchy of $16 / 14$, it showcases the crucial structures that we exploit later in this section when proving Theorem 1. 


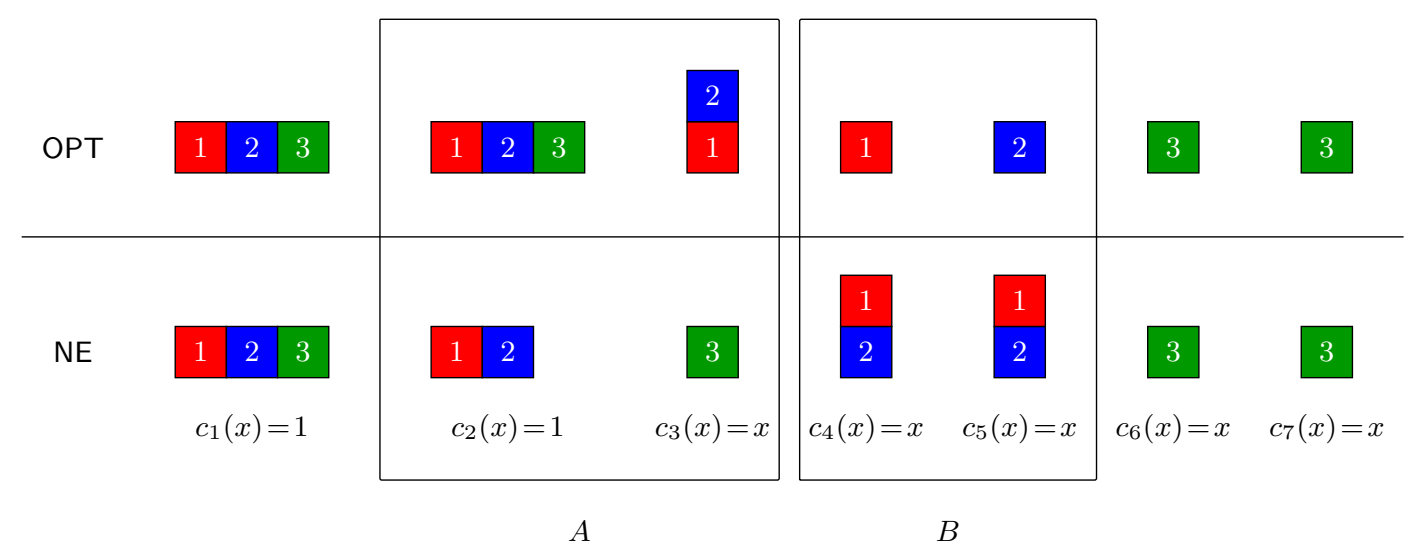

Figure 1 A symmetric 4-uniform matroid congestion game with seven resources. The hight of the stack of each resource corresponds to its cost.

- Example 2. Consider the symmetric 4-uniform matroid congestion game in Figure 1. There are seven resources $R=\{1, \ldots, 7\}$. The first two resources have constant cost functions $c_{1}(x)=c_{2}(x)=1$ for all $x \in \mathbb{N}$. The cost function of the other five resources is the identity, i.e., $c_{r}(x)=x$ for all $r \in\{3, \ldots, 7\}$. There are three players whose strategy is to choose exactly 4 resources, i.e., $X_{i}=\{S \subset R:|S|=4\}$ for all $i \in\{1,2,3\}$. In the system optimum, the two resources with constant costs are used by all players and each player chooses two of the remaining five resources, see the upper profile in Figure 1. One of the resources with non-constant costs has to be used by two players leading to overall costs of 14 . However, there is a Nash equilibrium, in which not all of the resources with constant costs are used by all players, see the lower profile in Figure 1. This Nash equilibrium has a total cost of 16 . The price of anarchy of this instance is $16 / 14 \approx 1.14$.

In order to derive bounds on the price of anarchy for the proof of Theorem 1, we bound the excess costs of the resources that are chosen by more players in the Nash equilibrium than in the system optimum in terms of the excess costs of the resources that are chosen by more players in the system optimum than in the Nash equilibrium. To this end, we denote by $A$ the set of resources chosen by more players in $x^{\mathrm{OPT}}$ than in $x^{\mathrm{NE}}$, and by $B$ the set of resources chosen by more players in $x^{\mathrm{NE}}$ than in $x^{\mathrm{OPT}}$, i.e.,

$$
A=\left\{r \in R: x_{r}^{\mathrm{OPT}}>x_{r}^{\mathrm{NE}}\right\} \quad \text { and } \quad B=\left\{r \in R: x_{r}^{\mathrm{OPT}}<x_{r}^{\mathrm{NE}}\right\} .
$$

Henceforth, we call the resources in $A$ are underdemanded and the resources in $B$ are overdemanded. For an illustration, see also Figure 1 where the set of underdemanded resources is $A=\{2,3\}$ and the set of overdemanded resources is $B=\{4,5\}$.

As we show in the following lemma, it is sufficient to bound the excess costs of the resources in $B$ in terms of the excess costs of of the resources in $A$ in order to bound the price of anarchy.

Lemma 3. For a symmetric $k$-uniform congestion game with affine cost functions and $A$ and $B$ as in (1), we have

$$
\frac{3}{4} C\left(x^{\mathrm{NE}}\right) \leq C\left(x^{\mathrm{OPT}}\right)+\sum_{b \in B}\left(x_{b}^{\mathrm{NE}}-x_{b}^{\mathrm{OPT}}\right) c_{b}\left(x_{b}^{\mathrm{NE}}\right)-\sum_{a \in A}\left(x_{a}^{\mathrm{OPT}}-x_{a}^{\mathrm{NE}}\right) c_{a}\left(x_{a}^{\mathrm{NE}}+1\right) .
$$

The proof is a rather straightforward generalization of a similar lemma due to Fotakis [11] for singleton games. It is contained in the appendix. 
In order to use Lemma 3 for the proof of Theorem 1, we are interested in bounding $\sum_{b \in B}\left(x_{b}^{\mathrm{NE}}-x_{b}^{\mathrm{OPT}}\right) c_{b}\left(x_{b}^{\mathrm{NE}}\right)-\sum_{a \in A}\left(x_{a}^{\mathrm{OPT}}-x_{a}^{\mathrm{NE}}\right) c_{a}\left(x_{a}^{\mathrm{NE}}+1\right)$ in terms of $C\left(x^{\mathrm{NE}}\right)$. It is interesting to note that for symmetric singleton games, it holds that

$$
c_{r}\left(x^{\mathrm{NE}}\right) \leq c_{r^{\prime}}\left(x_{r^{\prime}}^{\mathrm{NE}}+1\right)
$$

for all $r, r^{\prime} \in R$ by the Nash inequality. This implies in particular that

$$
\sum_{b \in B}\left(x_{b}^{\mathrm{NE}}-x_{b}^{\mathrm{OPT}}\right) c_{b}\left(x_{b}^{\mathrm{NE}}\right) \leq \sum_{a \in A}\left(x_{a}^{\mathrm{OPT}}-x_{a}^{\mathrm{NE}}\right) c_{a}\left(x_{a}^{\mathrm{NE}}+1\right),
$$

which together with Lemma 3 implies an upper bound on the price of anarchy of $4 / 3$. This is the road taken by Fotakis [11] in order to derive this bound.

However, neither inequality (3) nor inequality (4) hold in $k$-uniform matroid congestion games due to the more complicated strategy spaces. E.g., for the Nash equilibrium $x^{\mathrm{NE}}$ and system optimum $x^{\mathrm{OPT}}$ in Figure 1 we have $c_{4}\left(x_{4}^{\mathrm{NE}}\right)=2>c_{2}\left(x_{2}^{\mathrm{NE}}+1\right)=1$ as well as $c_{4}\left(x_{4}^{\mathrm{NE}}\right)+c_{5}\left(x_{5}^{\mathrm{NE}}\right)=4>3=c_{2}\left(x_{2}^{\mathrm{NE}}+1\right)+c_{3}\left(x_{2}^{\mathrm{NE}}+1\right)$. More generally speaking, inequality (4) does not hold if all players choosing an overloaded resource $b \in B$ in $x^{\mathrm{NE}}$ also choose an underloaded resource $a \in A$. The main technical work in our proof of Theorem 1 is be to derive an alternative upper bound for the right hand side in (2). Specifically, we will work towards showing that for $k$-uniform congestion games, we have

$$
\sum_{b \in B}\left(x_{b}^{\mathrm{NE}}-x_{b}^{\mathrm{OPT}}\right) c_{b}\left(x_{b}^{\mathrm{NE}}\right)-\sum_{a \in A}\left(x_{a}^{\mathrm{OPT}}-x_{a}^{\mathrm{NE}}\right) c_{a}\left(x_{a}^{\mathrm{NE}}+1\right) \leq \frac{2}{7} C\left(x^{\mathrm{NE}}\right) .
$$

In order to show inequality (5), some further notation is necessary. A natural way of decomposing the cost of a strategy vector $x$ is to consider the tuples $(i, r)$ with the property that player $i$ uses resource $r$ in strategy $x$. One may think of such a tuple as a single unit of demand that player $i$ places on resource $r$ under strategy vector $x$. The cost of a unit of demand is equal to the cost of the corresponding resource under that strategy profile, and the cost of strategy profile is then equal to the sum of the costs of the units of demand. Let

$$
P_{A} \subseteq\left\{(i, a): a \in A, a \in x_{i}^{\mathrm{NE}}\right\}
$$

be a subset of the units of demand placed in $x^{\mathrm{NE}}$ on the resources in $A$ such that

$$
\left|\left\{(i, a) \in P_{A}\right\}\right|=x_{a}^{\mathrm{OPT}}-x_{a}^{\mathrm{NE}} \quad \text { for all } a \in A,
$$

i.e., for each resource $a \in A, P_{A}$ contains exactly as many units of demand as there are more on these resources in $x^{\mathrm{OPT}}$ than in $x^{\mathrm{NE}}$. Similarly, let

$$
P_{B} \subseteq\left\{(i, b): b \in B, b \in x_{i}^{\mathrm{OPT}}\right\}
$$

be such that

$$
\left|\left\{(i, b) \in P_{B}\right\}\right|=x_{b}^{\mathrm{NE}}-x_{b}^{\mathrm{OPT}} \quad \text { for all } b \in B .
$$

Given these definitions, we want to bound the total costs of the units in $P_{B}$ with respect to the total costs of the units in $P_{A}$. We first identify a subset of these units, for which a simple bound can be obtained, i.e., we identify units of demand $(i, a) \in P_{A}$ and $(j, b) \in P_{B}$ such that $c_{b}\left(x_{b}^{\mathrm{NE}}\right) \leq c_{a}\left(x_{a}^{\mathrm{NE}}+1\right)$. For our purposes, it is sufficient to do this iteratively in a greedy way, see the greedy cancelling process in Algorithm 1. 


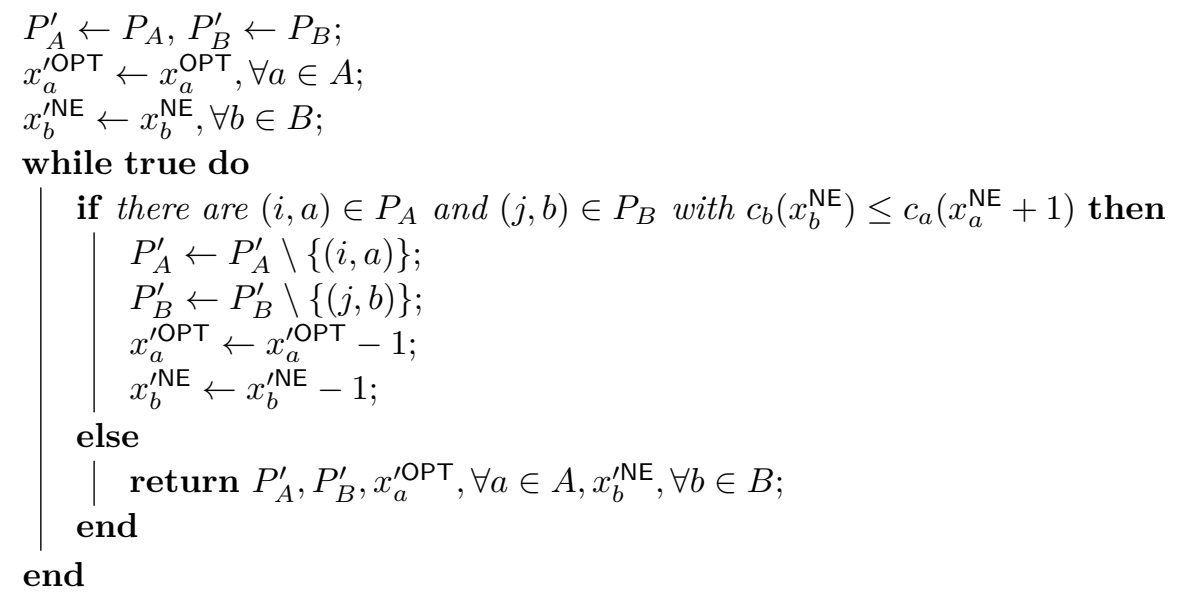

Algorithm 1: Cancelling process

Intuitively, this algorithm maps all units of demand in $P_{B}$ whose cost are bounded by the cost of another unit in $P_{A}$ and removes both units from the sets $P_{A}$ and $P_{B}$. In the following, we denote by $P_{A}^{\prime} \subseteq P_{A}$ and $P_{B}^{\prime} \subseteq P_{B}$ the set of units that survives this elimination. We denote by $x_{a}^{\prime \mathrm{OPT}}$ and $x_{b}^{\prime \mathrm{NE}}$ the number of units of demand that survive this elimination on each underdemanded and overdemanded resource respectively. Note that by definition of $P_{A}$ and $P_{B}$, we have that $x_{b}^{\prime \mathrm{NE}} \geq x_{b}^{\mathrm{OPT}}$ for $b \in B$, and $x_{a}^{\prime \mathrm{OPT}} \geq x_{a}^{\mathrm{NE}}$ for $a \in A$ after the cancelling.

Also note that during the course of the algorithm there may be different pairs $(i, a) \in P_{A}$ and $(j, b) \in P_{B}$ for which the condition in the if-loop is satisfied. For our following arguments it is irrelevant, which of these is removed from $P_{A}$ and $P_{B}$. Let

$$
\begin{aligned}
& A^{\prime}=\left\{a \in A: \text { there is }(i, a) \in P_{A}^{\prime} \text { for some } i \in N\right\}, \\
& B^{\prime}=\left\{b \in B: \text { there is }(i, b) \in P_{B}^{\prime} \text { for some } i \in N\right\}
\end{aligned}
$$

be the resources that remain over- respectively underdemanded in $x^{\mathrm{NE}}$ as opposed to $x^{\mathrm{OPT}}$ after the cancelling process. The following lemma then follows directly by definition of the above cancelling process and states that the cost of cancelled packets on $B$ with respect to $c_{b}\left(x_{b}^{\mathrm{NE}}\right)$ is bounded by the cost of the cancelled packets on $A$ with respect to $c_{a}\left(x_{a}^{\mathrm{NE}}+1\right)$.

Lemma 4. For a symmetric $k$-uniform congestion game with affine cost functions, $A$ and $B$ as in (1), and $A^{\prime}$ and $B^{\prime}$ as in (6), we have

$$
\sum_{b \in B}\left(x_{b}^{\mathrm{NE}}-x_{b}^{\prime \mathrm{NE}}\right) c_{b}\left(x_{b}^{\mathrm{NE}}\right)-\sum_{a \in A}\left(x_{a}^{\mathrm{OPT}}-x_{a}^{\prime \mathrm{OPT}}\right) c_{a}\left(x_{a}^{\mathrm{NE}}+1\right) \leq 0 .
$$

For the following arguments, it may be helpful to consult Figure 2 that shows the outcome of the cancelling process and the resulting sets $A^{\prime}$ and $B^{\prime}$ for the congestion game introduced in Example 2.

Let us define

$$
P=\left\{(i, r): r \in R, r \in x_{i}^{\mathrm{NE}}\right\}
$$

as the set of all units of demand in $x^{\mathrm{NE}}$. The next lemma is the first, crucial ingredient that allows us to obtain improved bounds on the price of anarchy. It states that for each overdemanded unit of demand on a resource in $P_{B}^{\prime}$, there are "enough" other units on other resources. Subsequently, we are also going to bound the cost of these other units from below. 


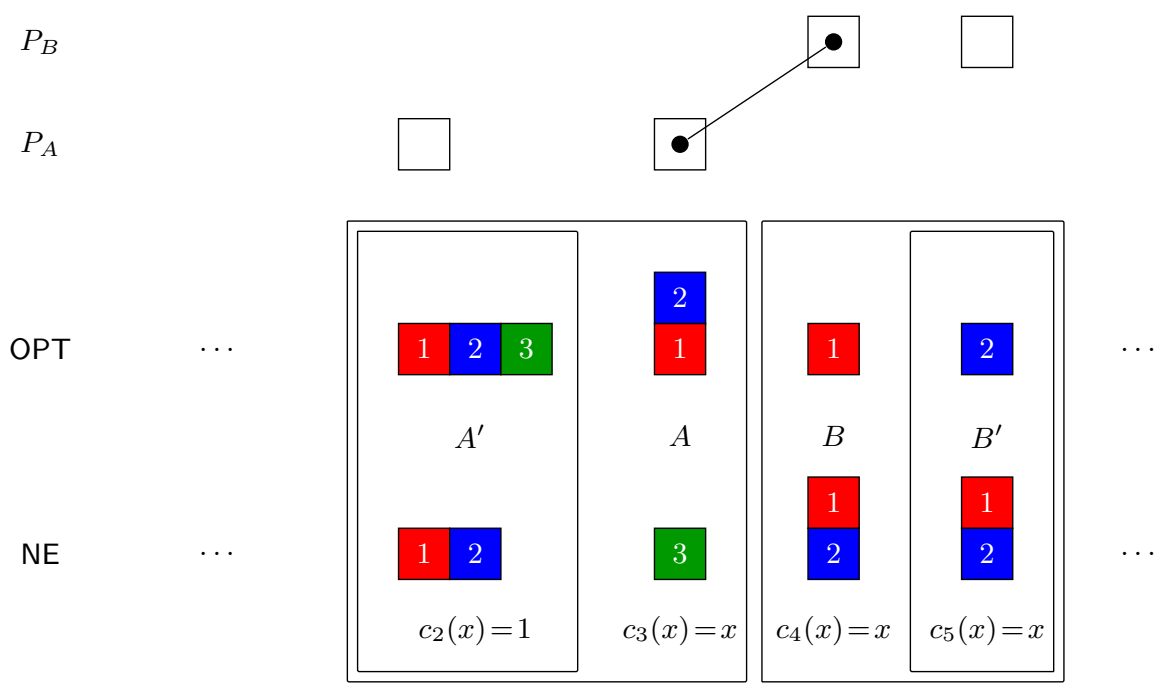

Figure 2 Underloaded resources $A=\{2,3\}$ and overloaded resources $B=\{4,5\}$ for the game considered in Example 2. In the cancelling process one unit of demand of resource 4 cancelled out with a unit of demand of resource 3 . After the cancelling process only resource 2 is underloaded and only resource 5 is overloaded, i.e., $A^{\prime}=\{2\}$ and $B^{\prime}=\{5\}$.

- Lemma 5. For a symmetric $k$-uniform congestion game with affine cost functions, let $P$ be as in (7) and let $\left(P_{A}^{\prime}, P_{B}^{\prime}\right)$ be the output of Algorithm 1. Then, $\left|P \backslash P_{B}^{\prime}\right| \geq 3\left|P_{B}^{\prime}\right|$.

Proof. To prove the lemma, we identify for each unit of demand $(i, b) \in P_{B}^{\prime}$ a set of at least three units of demand in $P \backslash P_{B}^{\prime}$.

To this end, let $l:=\left|A^{\prime}\right|$ and let $p$ be the maximum units of demand put by a single player on resources in $B^{\prime}$, i.e.

$$
p:=\max _{i \in N}\left|\left\{(i, b) \in P_{B}^{\prime}\right\}\right| .
$$

Note that there are at least $\left|P_{B}^{\prime}\right| / p$ players that have a unit of demand in $P_{B}^{\prime}$. Since, the units of demand in $P_{B}^{\prime}$ survived the cancelling process, and the cancelling process eliminates units of demand in $P_{B}$ together with units of demands in $P_{A}$ with $c_{b}\left(x_{b}^{\mathrm{NE}}\right) \leq c_{a}\left(x_{a}^{\mathrm{NE}}+1\right)$, we derive that $x^{\mathrm{NE}}$ can only be a Nash equilibrium if all players with a unit of demand in $P_{B}^{\prime}$ already use all resources in $A^{\prime}$. Put differently, for every $\left|P_{B}^{\prime}\right| / p$ units of demand in $P_{B}^{\prime}$, there are $l$ units of demand on resources in $A^{\prime}$, i.e., there are at least

$$
l \frac{\left|P_{B}^{\prime}\right|}{p}
$$

units of demand on resources in $A^{\prime}$ in $x^{\mathrm{NE}}$. The above argumentation further implies that $k \geq l+p$.

Further, there are $\left|P_{A}^{\prime}\right|$ units of demand that were put on resources in $A^{\prime}$ in $x^{\mathrm{OPT}}$, that are not there in $x^{\mathrm{NE}}$. This implies the existence of a set $N^{\prime}$ of players and $q_{i} \in\{1, \ldots, l\}$ for all $i \in N^{\prime}$ such that each player $i \in N^{\prime}$ uses $q_{i}$ resources in $A^{\prime}$ less in $x^{\mathrm{NE}}$ than in $x^{\mathrm{OPT}}$. For each player $i \in N^{\prime}$, let $q_{i}^{\prime} \leq l-1$ be the number of resources in $A^{\prime}$ still used in $x^{\mathrm{NE}}$. Each of these players uses $k$ resources in $x^{\mathrm{NE}}$, but at most $l-1$ resources among those in $A^{\prime}$. Thus, by the fact that $x^{\mathrm{NE}}$ is a Nash equilibrium and deviations from a resource in $B^{\prime}$ to a resource 
in $A^{\prime}$ are profitable, none of the players in $N^{\prime}$ uses a resource in $B^{\prime}$ in the Nash equilibrium. We obtain that there are at least

$$
\sum_{i \in N^{\prime}}\left(k-q_{i}^{\prime}\right)
$$

units of demand in $P \backslash\left(P_{B}^{\prime} \cup P_{A}^{\prime}\right)$ on resources in $R \backslash\left(A^{\prime} \cup B^{\prime}\right)$. We next bound (9) from below observing that $\sum_{i \in N^{\prime}} q_{i}^{\prime} \leq\left|N^{\prime}\right| l-\sum_{i \in N^{\prime}} q_{i}$, as $q_{i}+q_{i}^{\prime} \leq l$. Together with the fact that

$$
\left|N^{\prime}\right| \geq \frac{\left|P_{A}^{\prime}\right|}{l}=\frac{\left|P_{B}^{\prime}\right|}{l}
$$

we then obtain that there are at least

$$
\begin{aligned}
\sum_{i \in N^{\prime}}\left(k-q_{i}^{\prime}\right) & \geq\left|N^{\prime}\right|(k-l)+\sum_{i \in N^{\prime}} q_{i} \\
& =\left|N^{\prime}\right|(k-l)+\left|P_{B}^{\prime}\right| \\
& \geq \frac{\left|P_{B}^{\prime}\right|}{l}(k-l)+l \frac{\left|P_{B}^{\prime}\right|}{l}=k \frac{\left|P_{B}^{\prime}\right|}{l}
\end{aligned}
$$

units of demand in $x^{\mathrm{NE}}$ on resources in $R \backslash\left(A^{\prime} \cup B^{\prime}\right)$. Given that in (8) we only accounted for units of demand on resources in $A^{\prime}$, and in (10) we only accounted for units of demand on resources in $R \backslash\left(A^{\prime} \cup B^{\prime}\right)$, we do not count any units of demand twice when lower bounding the number of units $\left|P \backslash P_{B}^{\prime}\right|$ by

$$
l \frac{\left|P_{B}^{\prime}\right|}{p}+k \frac{\left|P_{B}^{\prime}\right|}{l} \geq l \frac{\left|P_{B}^{\prime}\right|}{p}+(l+p) \frac{\left|P_{B}^{\prime}\right|}{l}=\left|P_{B}^{\prime}\right|\left(1+\frac{l}{p}+\frac{p}{l}\right) \geq 3\left|P_{B}^{\prime}\right|,
$$

which establishes the result.

Before we proceed, we provide two structural lemmas which restrict the space of instances with worst-case price of anarchy.

- Lemma 6. The worst-case price of anarchy of symmetric $k$-uniform congestion games is attained on games that have the property that no resource is chosen by all players both in an optimal strategy vector and a worst-case Nash equilibrium.

The proof is by contradiction and can be found in the appendix. The next lemma is a technical lemma specifically about the structure of worst-case instances with $\operatorname{PoA}(I) \geq 4 / 3$. Again, the proof is given in the appendix.

- Lemma 7. For any instance I of a symmetric $k$-uniform matroid congestion game with affine cost functions and $\mathrm{PoA}(I) \geq 4 / 3$ and a resource $r \in R \backslash B^{\prime}$, chosen by all players in $x^{\mathrm{NE}}$, there exists an instance $\tilde{I}$, with resource $r$ removed, such that $\operatorname{PoA}(\tilde{I}) \geq \operatorname{PoA}(I)$.

The restrictions on the structure of worst-case instances obtained in Lemma 7 will be used later in the proof of Theorem 1. Before we can do that, however, we proceed to bound the costs of the resources in $A^{\prime}$ with the following two lemmas.

- Lemma 8. For a symmetric k-uniform congestion game with affine cost functions, we have $c_{r}\left(x_{r}^{\mathrm{NE}}\right) \leq 2 c_{r^{\prime}}\left(x_{r^{\prime}}^{\mathrm{NE}}+1\right)$ for any two resources $r, r^{\prime}$, where $x_{r}^{\mathrm{NE}} \geq 1$ and $x_{r^{\prime}}^{\mathrm{NE}}<n$.

Proof. Let $r, r^{\prime}$ be as in the statement of the lemma. Let us assume for contradiction that $c_{r}\left(x_{r}^{\mathrm{NE}}\right)>2 c_{r^{\prime}}\left(x_{r^{\prime}}^{\mathrm{NE}}+1\right)$. Consider the set of resources

$$
R^{-}=\left\{r^{-} \in R: c_{r^{-}}\left(x_{r^{-}}^{\mathrm{NE}}\right) \leq c_{r^{\prime}}\left(x_{r^{\prime}}^{\mathrm{NE}}+1\right)\right\} .
$$


For each $r^{-} \in R^{-}$, we have

$$
c_{r^{-}}\left(x_{r^{-}}^{\mathrm{NE}}+1\right) \leq 2 c_{r^{-}}\left(x_{r^{-}}^{\mathrm{NE}}\right) \leq 2 c_{r^{\prime}}\left(x_{r^{\prime}}^{\mathrm{NE}}+1\right)<c_{r}\left(x_{r}^{\mathrm{NE}}\right) .
$$

Since $x^{\mathrm{NE}}$ is a Nash equilibrium, no player can improve by choosing a resource $r^{-} \in R^{-}$ instead of $r$. Thus, all players using $r$ in $x^{\mathrm{NE}}$ must also use all resources $r^{-} \in R^{-}$. This implies in particular that $\left|R^{-}\right|<k$. As any player chooses exactly $k$ resources, she chooses at least one resource $\tilde{r} \in R \backslash R^{-}$. By definition of $R^{-}, c_{\tilde{r}}\left(x_{\tilde{r}}^{\mathrm{NE}}\right)>c_{r^{\prime}}\left(x_{r^{\prime}}^{\mathrm{NE}}+1\right)$. Again, by the Nash property, this implies that all players are using $r^{\prime}$, so $x_{r^{\prime}}^{\mathrm{NE}}=n$, which contradicts the assumption that $x_{r^{\prime}}^{\mathrm{NE}}<n$.

- Lemma 9. For a symmetric $k$-uniform congestion game with affine cost functions, we have

$$
\sum_{b \in B^{\prime}}\left(x_{b}^{\mathrm{NE}}-x_{b}^{\mathrm{OPT}}\right) c_{b}\left(x_{b}^{\mathrm{NE}}\right) \leq 2 \sum_{a \in A^{\prime}}\left(x_{a}^{\prime \mathrm{OPT}}-x_{a}^{\mathrm{NE}}\right) c_{a}\left(x_{a}^{\mathrm{NE}}+1\right) .
$$

Proof. First recall that $x_{b}^{\mathrm{NE}} \geq 1$ for all $b \in B^{\prime}$, and $x_{a}^{\mathrm{NE}}<n$ for all $a \in A^{\prime}$ as resources in $A^{\prime} \subseteq A$ are chosen more often in $x^{\mathrm{OPT}}$ than in $x^{\mathrm{NE}}$. By Lemma 8 , we can therefore conclude that $c_{b}\left(x_{b}^{\mathrm{NE}}\right) \leq 2 c_{a}\left(x_{a}^{\mathrm{NE}}+1\right)$ for all resources $b \in B^{\prime}$ and $a \in A^{\prime}$. Summing over all units of demands in $P_{A}^{\prime}$ and $P_{B}^{\prime}$, respectively, yields the result.

We are now ready to prove our main theorem (Theorem 1).

Proof of Theorem 1. By Lemma 5, for each unit of demand in $P_{B}^{\prime}$, there are three distinct units of demand in $x^{\mathrm{NE}}$ on resources $r \in R \backslash B^{\prime}$. We bound the cost of each of these resource units from below by

$$
c_{r}\left(x_{r}^{\mathrm{NE}}\right) \geq \frac{c_{r}\left(x_{r}^{\mathrm{NE}}+1\right)}{2} \geq \frac{c_{b}\left(x_{b}^{\mathrm{NE}}\right)}{4},
$$

for any $b \in B$. Here the first inequality follows directly from the fact that the cost functions are affine. The second inequality follows from Lemma 8 for resources $r$ with $x_{r}^{\mathrm{NE}}<n$. However, by Lemma 7, it is without loss of generality to assume that no resource $r \in R \backslash B^{\prime}$ is chosen by all players in $x^{\mathrm{NE}}$, unless the price of anarchy is not larger than $4 / 3$. Therefore, we finally get

$$
\begin{aligned}
C\left(x^{\mathrm{NE}}\right) & \geq \sum_{b \in B^{\prime}}\left(x_{b}^{\mathrm{NE}}-x_{b}^{\mathrm{OPT}}\right) c_{b}\left(x_{b}^{\mathrm{NE}}\right)+\sum_{b \in B^{\prime}} x_{b}^{\mathrm{OPT}} c_{b}\left(x_{b}^{\mathrm{NE}}\right)+\sum_{r \in R \backslash B^{\prime}} x_{r}^{\mathrm{NE}} c_{r}\left(x_{r}^{\mathrm{NE}}\right) \\
& \geq \sum_{b \in B^{\prime}}\left(x_{b}^{\prime \mathrm{NE}}-x_{b}^{\mathrm{OPT}}\right) c_{b}\left(x_{b}^{\mathrm{NE}}\right)+\sum_{r \in R \backslash B^{\prime}} x_{r}^{\mathrm{NE}} c_{r}\left(x_{r}^{\mathrm{NE}}\right) \\
& \geq \frac{7}{4} \sum_{b \in B^{\prime}}\left(x_{b}^{\prime \mathrm{NE}}-x_{b}^{\mathrm{OPT}}\right) c_{b}\left(x_{b}^{\mathrm{NE}}\right) .
\end{aligned}
$$

Here, the first inequality uses $x_{b}^{\prime N E} \leq x_{b}^{\mathrm{NE}}$ for any resource $b \in B$, which follows from the cancelling process. The last inequality uses that $\sum_{b \in B^{\prime}}\left(x_{b}^{\prime \mathrm{NE}}-x_{b}^{\mathrm{OPT}}\right)=\left|P_{B}^{\prime}\right|$, and by Lemma 5 , $\sum_{r \in R \backslash B^{\prime}} x_{r}^{\mathrm{NE}} \geq\left|P \backslash P_{B}^{\prime}\right| \geq 3\left|P_{B}^{\prime}\right|=3 \sum_{b \in B^{\prime}}\left(x_{b}^{\prime \mathrm{NE}}-x_{b}^{\mathrm{OPT}}\right)$, and each of these resource units has cost at least $c_{b}\left(x_{b}^{\mathrm{NE}}\right) / 4$, for all $b \in B$ by (11). 
Combining (12) with Lemma 4 and Lemma 9 yields

$$
\begin{aligned}
& \sum_{b \in B}\left(x_{b}^{\mathrm{NE}}-x_{b}^{\mathrm{OPT}}\right) c_{b}\left(x_{b}^{\mathrm{NE}}\right)-\sum_{a \in A}\left(x_{a}^{\mathrm{OPT}}-x_{a}^{\mathrm{NE}}\right) c_{a}\left(x_{a}^{\mathrm{NE}}+1\right) \\
\leq & \sum_{b \in B^{\prime}}\left(x_{b}^{\mathrm{NE}}-x_{b}^{\mathrm{OPT}}\right) c_{b}\left(x_{b}^{\mathrm{NE}}\right)-\sum_{a \in A^{\prime}}\left(x_{a}^{\mathrm{OPT}}-x_{a}^{\mathrm{NE}}\right) c_{a}\left(x_{a}^{\mathrm{NE}}+1\right) \\
\leq & \frac{1}{2} \sum_{b \in B^{\prime}}\left(x_{b}^{\prime \mathrm{NE}}-x_{b}^{\mathrm{OPT}}\right) c_{b}\left(x_{b}^{\mathrm{NE}}\right) \\
\leq & \frac{2}{7} C\left(x^{\mathrm{NE}}\right)
\end{aligned}
$$

where the first inequality is by Lemma 4, the second by Lemma 9, and the third by (12). Finally, plugging this into (2) proves Theorem 1.

\section{$4 \quad$ Lower Bound}

In this section, we show that generalizing the strategy spaces from singletons to $k$-uniform matroids increases the price of anarchy of congestion games.

- Theorem 10. The price of anarchy of symmetric $k$-uniform congestion games with affine cost functions is at least $7-4 \sqrt{2} \approx 1.343$ for large enough $k$.

Proof. We first give a lower bound on the price of anarchy of $47 / 35 \approx 1.3428$, and then we explain how to extend the example to construct a series of examples for which the price of anarchy converges to $7-4 \sqrt{2}>1.343$.

The set of resources is partitioned into 3 sets $U, V$, and $W$. The set $U$ consists of two resources with constant cost equal to 1 , the set $V$ consists of 6 resources and $W$ consists of 21 resources. Each resource $r \in V \cup W$ has costs equal to the number of players using it, i.e., $c_{r}\left(x_{r}\right)=x_{r}$. There are seven players. Any set which consists of exactly 5 resources is a feasible strategy.

A system optimum solution and a Nash equilibrium for this game is shown in Figure 3 . In the Nash equilibrium, players 5, 6 and 7 each choose five resources from set $W$ and pay in total a cost of 5 . The four other players each choose two resources from $U$ and share three resources from $V$ with one other player. Note that none of these players can improve by deviating; all of them already use all resources in $U$, and all resources $V \cup W$ cost at least 2 after a deviation. This equilibrium yields a total cost of $4 \times 2+15+6 \times 2 \times 2=47$. In the system optimal strategy vector, each player chooses 2 resources in $U$, and 3 resources in $V \cup W$ such that none of the resources in $V \cup W$ is used by more than one player. This yields a total cost of $7 \times 5=35$. We conclude that for this instance the price of anarchy is equal to $47 / 35 \approx 1.3428$.

We can slightly improve this bound by optimizing over the number of resources contained in the sets $U, V$, and $W$. To this end, for some integers $p, q \in \mathbb{N}$, let the set of resources be partitioned into three sets $U, V$, and $W$ where $U$ consists of $q$ resources with constant cost 1 , $V$ consists of $p \cdot q$ resources, and $W$ consists of $p(p+q)$ resources. As before, each resource $r$ in $V$ or $W$ has cost equal to the number of players choosing it, i.e. $c_{r}\left(x_{r}\right)=x_{r}$. There are $p+2 q$ players. Any set which consists of exactly $p+q$ resources is a feasible strategy. Analogously to the example above, there is a Nash equilibrium where $p$ players choose $p+q$ resources from $W$ (yielding cost $p+q$ for these players), and $2 q$ players choose $q$ resources from $U$ and share $p$ resources from $V$ with one other player. As before, none of these players can improve by deviating; no more resources in $U$ can be chosen, and all resources in $V \cup W$ 

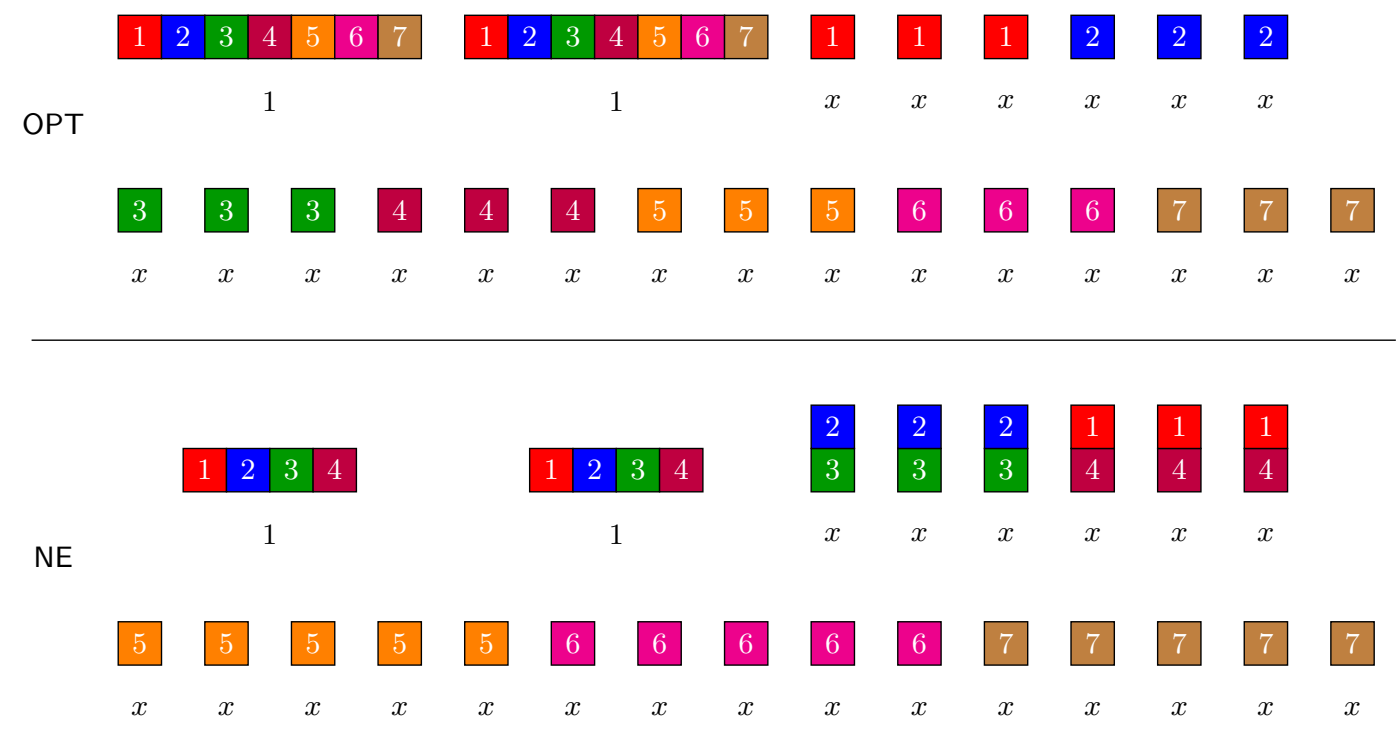

Figure 3 A lower bound instance. Stacks and lines of packets correspond to resources. In the system optimal solution, every resource is used by exactly one player while in the Nash equilibrium six of the resources are used by 2 players each.

cost at least 2. This equilibrium yields a total cost of $p(p+q)+2 q(q+2 p)=p^{2}+5 p q+2 q^{2}$. In the system optimum, each player chooses $q$ resources in $U$, and $p$ free resources in $V \cup W$, yielding a total cost of $(p+2 q)(p+q)=p^{2}+3 p q+2 q^{2}$. This implies for the price of anarchy of $k$-uniform congestion games with affine costs that

$$
\mathrm{PoA} \geq \sup _{p, q \in \mathbb{N}} \frac{p^{2}+5 p q+2 q^{2}}{p^{2}+3 p q+2 q^{2}}=\sup _{p, q \in \mathbb{N}} \frac{\left(\frac{p}{q}\right)^{2}+5 \frac{p}{q}+2}{\left(\frac{p}{q}\right)^{2}+3 \frac{p}{q}+2}=\max _{x \in \mathbb{R} \geq 0} \frac{x^{2}+5 x+2}{x^{2}+3 x+2} .
$$

Basic calculus shows that this maximum is attained at $x=\sqrt{2}$, yielding PoA $\geq 7-4 \sqrt{2}$. For $p, q \in \mathbb{Z}$, we can get arbitrarily close to this value. In our first example $p=3$ and $q=2$.

\section{Conclusions}

The most interesting open problem, next to improving lower and upper bounds for the $k$-uniform matroid case we study here, is to analyze the price of anarchy for the generalized problem with arbitrary matroid strategy spaces.

\section{References}

1 Fidaa Abed, José R. Correa, and Chien-Chung Huang. Optimal coordination mechanisms for multi-job scheduling games. In A. Schulz and D. Wagner, editors, Proc. 22th Annu. European Symp. Algorithms, volume 8737 of LNCS, pages 13-24, 2014.

2 Heiner Ackermann, Heiko Röglin, and Berthold Vöcking. On the impact of combinatorial structure on congestion games. J. ACM, 55(6):1-22, 2008.

3 Heiner Ackermann, Heiko Röglin, and Berthold Vöcking. Pure Nash equilibria in playerspecific and weighted congestion games. Theoret. Comput. Sci., 410(17):1552-1563, 2009.

4 Sebastian Aland, Dominic Dumrauf, Martin Gairing, Burkhard Monien, and Florian Schoppmann. Exact price of anarchy for polynomial congestion games. SIAM J. Comput., 40(5):1211-1233, 2011. 
5 Baruch Awerbuch, Yossi Azar, and Amir Epstein. The price of routing unsplittable flow. In Proc. 37th Annu. ACM Symp. Theory Computing, pages 57-66, 2005.

6 Martin Beckmann, C. B. McGuire, and Christopher B. Winsten. Studies in the Economics and Transportation. Yale University Press, New Haven, CT, USA, 1956.

7 Dietrich Braess. Über ein Paradoxon aus der Verkehrsplanung. Unternehmensforschung, 12:258-0268, 1968. (German).

8 Ioannis Caragiannis, Michele Flammini, Christos Kaklamanis, Panagiotis Kanellopoulos, and Luca Moscardelli. Tight bounds for selfish and greedy load balancing. In M. Bugliesi, B. Preneel, V. Sassone, and I. Wegener, editors, Proc. 33rd Int. Colloquium Automata, Languages and Programming, volume 4052 of LNCS, pages 184-195, 2006.

9 George Christodoulou and Elias Koutsoupias. The price of anarchy of finite congestion games. In Proc. 37th Annu. ACM Symp. Theory Computing, pages 67-73, 2005.

10 Juliane Dunkel and Andreas S. Schulz. On the complexity of pure-strategy Nash equilibria in congestion and local-effect games. Math. Oper. Res., 33(4):851-868, 2008.

11 Dimitris Fotakis. Stackelberg strategies for atomic congestion games. Theory Comput. Syst., 47:218-249, 2010.

12 Satoru Fujishige, Michel X. Goemans, Tobias Harks, Britta Peis, and Rico Zenklusen. Matroids are immune to Braess paradox. arXiv:1504.07545, 2015.

13 Martin Gairing, Thomas Lücking, Marios Mavronicolas, Burkhard Monien, and Manuel Rode. Nash equilibria in discrete routing games with convex latency functions. In J. Díaz, J. Karhumäki, A. Lepistö, and D. Sannella, editors, Proc. 31st Int. Colloquium Automata, Languages and Programming, volume 3142 of LNCS, pages 645-657, 2004.

14 Martin Gairing and Florian Schoppmann. Total latency in singleton congestion games. In X. Deng and F. Graham, editors, Proc. 3rd Int. Workshop Internet and Network Econ., volume 4858 of $L N C S$, pages 381-387, 2008.

15 Michel X. Goemans, Vahab S. Mirrokni, and Adrian Vetta. Sink equilibria and convergence. In Proc. 46th Annu. IEEE Symp. Found. Comput. Sci., pages 142-154, 2005.

16 Tobias Harks, Max Klimm, and Britta Peis. Resource competition on integral polymatroids. In Tie-Yan Liu, Qi Qi, and Yinyu Ye, editors, Proc. 10th Int. Conf. Web and Internet Econ., volume 8877 of $L N C S$, pages 189-202, 2014.

17 Tobias Harks and Britta Peis. Resource buying games. Algorithmica, 70(3):493-512, 2014.

18 Elias Koutsoupias and Christos Papadimitriou. Worst-case equilibria. In C. Meinel and S. Tison, editors, Proc. 16th Annu. Sympos. Theoretical Aspects Comput. Sci., volume 1563 of $L N C S$, pages 404-413, 1999.

19 Thomas Lücking, Marios Mavronicolas, Burkhard Monien, and Manuel Rode. A new model for selfish routing. Theoret. Comput. Sci., 406(3):187-206, 2008.

20 Carol Meyers. Network Flow Problems and Congestion Games: Complexity and Approximation Results. PhD thesis, MIT, Operations Research Center, 2006.

21 Igal Milchtaich. Congestion games with player-specific payoff functions. Games Econom. Behav., 13(1):111-124, 1996.

22 Arthur C. Pigou. The Economics of Welfare. Macmillan, London, UK, 1920.

23 Robert W. Rosenthal. A class of games possessing pure-strategy Nash equilibria. Internat. J. Game Theory, 2(1):65-67, 1973.

24 Robert W. Rosenthal. The network equilibrium problem in integers. Networks, 3:53-59, 1973.

25 Tim Roughgarden. The price of anarchy is independent of the network topology. J. Comput. System Sci., 67:341-364, 2002.

26 Tim Roughgarden and Éva Tardos. How bad is selfish routing? J. ACM, 49(2):236-259, 2002 . 
27 Subhash Suri, Csaba D. Tóth, and Yunhong Zhou. Selfish load balancing and atomic congestion games. Algorithmica, 47(1):79-96, 2007.

28 Long Tran-Thanh, Maria Polukarov, Archie Chapman, Alex Rogers, and Nicholas R. Jennings. On the existence of pure strategy Nash equilibria in integer-splittable weighted congestion games. In G. Persiano, editor, Proc. 4th Int. Symp. Algorithmic Game Theory, volume 6982 of $L N C S$, pages 236-253, 2011.

29 John G. Wardrop. Some theoretical aspects of road traffic research. Proceedings of the Institution of Civil Engineers, 1(3):325-362, 1952.

\section{A Omitted Proofs}

Proof of Lemma 3. For any resource $a \in A$ we have that

$$
\begin{aligned}
x_{a}^{\mathrm{NE}} c_{a}\left(x_{a}^{\mathrm{NE}}\right) & =x_{a}^{\mathrm{OPT}} c_{a}\left(x_{a}^{\mathrm{OPT}}\right)-x_{a}^{\mathrm{OPT}} c_{a}\left(x_{a}^{\mathrm{OPT}}\right)+x_{a}^{\mathrm{NE}} c_{a}\left(x_{a}^{\mathrm{NE}}\right) \\
& \leq x_{a}^{\mathrm{OPT}} c_{a}\left(x_{a}^{\mathrm{OPT}}\right)-x_{a}^{\mathrm{OPT}} c_{a}\left(x_{a}^{\mathrm{NE}}+1\right)+x_{a}^{\mathrm{NE}} c_{a}\left(x_{a}^{\mathrm{NE}}+1\right) \\
& =x_{a}^{\mathrm{OPT}} c_{a}\left(x_{a}^{\mathrm{OPT}}\right)-\left(x_{a}^{\mathrm{OPT}}-x_{a}^{\mathrm{NE}}\right) c_{a}\left(x_{a}^{\mathrm{NE}}+1\right)
\end{aligned}
$$

The second inequality uses that $x_{a}^{\mathrm{NE}}+1 \leq x_{a}^{\mathrm{OPT}}$ by the definition of $A$, and that the cost-functions are non-decreasing. For any resource $b \in B$, we have

$$
\begin{aligned}
x_{b}^{\mathrm{NE}} c_{b}\left(x_{b}^{\mathrm{NE}}\right) & =x_{b}^{\mathrm{OPT}} c_{b}\left(x_{b}^{\mathrm{NE}}\right)+\left(x_{b}^{\mathrm{NE}}-x_{b}^{\mathrm{OPT}}\right) c_{b}\left(x_{b}^{\mathrm{NE}}\right) \\
& \leq x_{b}^{\mathrm{OPT}} c_{b}\left(x_{b}^{\mathrm{OPT}}\right)+\frac{1}{4} x_{b}^{\mathrm{NE}} c_{b}\left(x_{b}^{\mathrm{NE}}\right)+\left(x_{b}^{\mathrm{NE}}-x_{b}^{\mathrm{OPT}}\right) c_{b}\left(x_{b}^{\mathrm{NE}}\right)
\end{aligned}
$$

Here, the inequality uses that the cost function is of the form $c_{b}(x)=\alpha x+\beta$ with $\alpha, \beta \geq 0$ and, thus,

$$
\begin{aligned}
x_{b}^{\mathrm{OPT}} c_{b}\left(x_{b}^{\mathrm{NE}}\right) & =\alpha x_{b}^{\mathrm{OPT}} x_{b}^{\mathrm{NE}}+\beta x_{b}^{\mathrm{OPT}} \\
& \leq \alpha x_{b}^{\mathrm{OPT}} x_{b}^{\mathrm{OPT}}+\beta x_{b}^{\mathrm{OPT}}+\frac{1}{4} \alpha x_{b}^{\mathrm{NE}} x_{b}^{\mathrm{NE}} \\
& \leq x_{b}^{\mathrm{OPT}} c_{b}\left(x_{b}^{\mathrm{OPT}}\right)+\frac{1}{4} x_{b}^{\mathrm{NE}} c_{b}\left(x_{b}^{\mathrm{NE}}\right),
\end{aligned}
$$

since

$$
0 \leq\left(x_{b}^{\mathrm{OPT}}-\frac{x_{b}^{\mathrm{NE}}}{2}\right)^{2}=\left(x_{b}^{\mathrm{OPT}}\right)^{2}+\left(\frac{x_{b}^{\mathrm{NE}}}{2}\right)^{2}-x_{b}^{\mathrm{OPT}} x_{b}^{\mathrm{NE}}
$$

for all $b \in B$. Finally, for any resource $r \in R \backslash(A \cup B)$ we have that

$$
x_{b}^{\mathrm{NE}} c_{b}\left(x_{b}^{\mathrm{NE}}\right)=x_{b}^{\mathrm{OPT}} c_{b}\left(x_{b}^{\mathrm{OPT}}\right)
$$

Summing over all resources yields:

$$
\begin{aligned}
c\left(x^{\mathrm{NE}}\right)= & \sum_{a \in A} x_{a}^{\mathrm{NE}} c_{a}\left(x_{a}^{\mathrm{NE}}\right)+\sum_{b \in B} x_{b}^{\mathrm{NE}} c_{b}\left(x_{b}^{\mathrm{NE}}\right)+\sum_{r \in R \backslash(A \cup B)} x_{r}^{\mathrm{NE}} c_{r}\left(x_{r}^{\mathrm{NE}}\right) \\
= & \sum_{r \in R} x_{r}^{\mathrm{OPT}} c_{r}\left(x_{r}^{\mathrm{OPT}}\right)+\frac{1}{4} \sum_{b \in B} x_{b}^{\mathrm{NE}} c_{b}\left(x_{b}^{\mathrm{NE}}\right) \\
& +\sum_{b \in B}\left(x_{b}^{\mathrm{NE}}-x_{b}^{\mathrm{OPT}}\right) c_{b}\left(x_{b}^{\mathrm{NE}}\right)-\sum_{a \in A}\left(x_{a}^{\mathrm{OPT}}-x_{a}^{\mathrm{NE}}\right) c_{a}\left(x_{a}^{\mathrm{NE}}+1\right) \\
\leq & C\left(x^{\mathrm{OPT}}\right)+\frac{1}{4} C\left(x^{\mathrm{NE}}\right)+\sum_{b \in B}\left(x_{b}^{\mathrm{NE}}-x_{b}^{\mathrm{OPT}}\right) c_{b}\left(x_{b}^{\mathrm{NE}}\right) \\
& -\sum_{a \in A}\left(x_{a}^{\mathrm{OPT}}-x_{a}^{\mathrm{NE}}\right) c_{a}\left(x_{a}^{\mathrm{NE}}+1\right)
\end{aligned}
$$


which establishes the claimed result.

Proof of Lemma 6 . Let $I$ be an arbitrary symmetric $k$-uniform congestion game, let $x^{\mathrm{NE}}$ be an arbitrary Nash equilibrium of $I$ maximizing $C(\cdot)$ and let $x^{\mathrm{OPT}}$ be an arbitrary strategy vector minimizing $C(\cdot)$.

Assume there is a resource $r$ used by all players in both $x^{\mathrm{NE}}$ and $x^{\mathrm{OPT}}$. We show that resource $r$ can be removed without decreasing the price of anarchy. Let $\tilde{I}$ be the symmetric $(k-1)$-uniform congestion game that has the same set of players as $I$, but the set of resources is reduced by $r$. Consider the strategy vector $\tilde{x}^{\mathrm{NE}}$ in $\tilde{I}$ where every player chooses the same resources as in $x^{\mathrm{NE}}$ except for $r$. Then $\tilde{x}^{\mathrm{NE}}$ is a Nash equilibrium in $\tilde{I}$. This implies that the Nash equilibrium $\tilde{x}^{\mathrm{NE}}$ with highest cost in $\tilde{I}$ has cost

$$
C\left(\tilde{x}^{\mathrm{NE}}\right) \geq C\left(x^{\mathrm{NE}}\right)-c_{r}(n) n
$$

Moreover, let $\tilde{x}^{\mathrm{OPT}}$ be the strategy vector of $\tilde{I}$ where all players choose the same resources as in $x^{\mathrm{OPT}}$, except for $r$. Then the optimality of $x^{\mathrm{OPT}}$ for $I$ yields that $\tilde{x}^{\mathrm{OPT}}$ must be optimal for $\tilde{I}$.

We have thus established that

$$
\operatorname{PoA}(I)=\frac{C\left(x^{\mathrm{NE}}\right)}{C\left(x^{\mathrm{OPT}}\right)} \leq \frac{C\left(\tilde{x}^{\mathrm{NE}}\right)+c_{r}(n)}{C\left(\tilde{x}^{\mathrm{OPT}}\right)+c_{r}(n)} \leq \operatorname{PoA}(\tilde{I}) .
$$

Successive elimination of all such resources $r$ yields the claimed result.

Proof of Lemma 7. Consider an instance $I$ with resource $r \in R \backslash B^{\prime}$ that is chosen by all players in a worst-case Nash equilibrium $x^{\mathrm{NE}}$. By Lemma 6 , we may assume that $r$ is not chosen by all players in $x^{\mathrm{OPT}}$, which implies $x_{r}^{\mathrm{NE}}>x_{r}^{\mathrm{OPT}}$ and, hence, $r \in B$.

Since $r$ is not in $B^{\prime}, q=x_{r}^{\mathrm{NE}}-x_{r}^{\mathrm{OPT}}$ units of demand on $r$ were canceled out during the cancelling process described in Algorithm 1. This implies the existence of $q$ resources $r_{1}, \ldots, r_{q}$ with $c_{r_{i}}\left(x_{r_{i}}^{\mathrm{OPT}}\right) \geq c_{r_{i}}\left(x_{r_{i}}^{\mathrm{NE}}+1\right) \geq c_{r}\left(x_{r}^{\mathrm{NE}}\right)$ for all $i \in\{1, \ldots, q\}$. The first inequality follows from $r_{i} \in A$ for all $i \in\{1, \ldots, q\}$. The second inequality follows from the canceling procedure.

Analogously to the proof Lemma 6 , let $\tilde{I}$ be the symmetric $(k-1)$-uniform congestion games with resource $r$ removed. The strategy vector $\tilde{x}^{\mathrm{NE}}$ in $\tilde{I}$ where every player chooses the same resources as in $x^{\mathrm{NE}}$, except for $r$, is a Nash equilibrium for $\tilde{I}$. It has cost $C\left(x^{\mathrm{NE}}\right)-c_{r}(n) n$. This implies that the Nash equilibrium $\tilde{x}^{\mathrm{NE}}$ with highest cost in $\tilde{I}$ has cost

$$
C\left(\tilde{x}^{\mathrm{NE}}\right) \geq C\left(x^{\mathrm{NE}}\right)-c_{r}(n) n
$$

We proceed to argue about the cost of an optimal strategy vector for $\tilde{I}$. To this end, note that it is feasible to remove one unit of demand for each player from $x^{\mathrm{OPT}}$. For all players $i$ with $r \in x_{i}^{\text {OPT }}$, we may simply remove the unit of demand put on $r$ (since $r$ is removed anyways). For all other players, by the cancelling process, there is a resource $r_{i}$, $i \in\{1, \ldots, q\}$ with $\operatorname{costs} c_{r_{i}}\left(x_{r_{i}}^{\mathrm{OPT}}\right) \geq x_{r}^{\mathrm{NE}}$, so we may remove the unit of demand put on this resource. This yields a feasible solution, and therefore the cost of an optimal strategy vector $\tilde{x}^{\mathrm{OPT}}$ for $\tilde{I}$ is at most

$$
\begin{aligned}
C\left(\tilde{x}^{\mathrm{OPT}}\right) & \leq C\left(x^{\mathrm{OPT}}\right)-x_{r}^{\mathrm{OPT}} c_{r}\left(x_{r}^{\mathrm{OPT}}\right)-\sum_{i=1, \ldots, q} c_{r_{i}}\left(x_{r_{i}}^{\mathrm{OPT}}\right) \\
& \leq C\left(x^{\mathrm{OPT}}\right)-x_{r}^{\mathrm{OPT}} c_{r}\left(x_{r}^{\mathrm{OPT}}\right)-\left(n-x_{r}^{\mathrm{OPT}}\right) c_{r}(n) \\
& \leq C\left(x^{\mathrm{OPT}}\right)-\frac{3}{4} c_{r}(n) n .
\end{aligned}
$$


Note that the last inequality follows by basic algebra because of the fact that the cost functions are affine. Inequalities (14) and (15) together imply that

$$
\operatorname{PoA}(\tilde{I}) \geq \frac{C\left(x^{\mathrm{NE}}\right)-c_{r}(n) n}{C\left(x^{\mathrm{OPT}}\right)-\frac{3}{4} c_{r}(n) n}=\frac{C\left(x^{\mathrm{NE}}\right)-c_{r}(n) n}{\frac{1}{\operatorname{PoA}(I)} C\left(x^{\mathrm{NE}}\right)-\frac{3}{4} c_{r}(n) n} \geq \frac{C\left(x^{\mathrm{NE}}\right)-c_{r}(n) n}{\frac{C\left(x^{\mathrm{NE}}\right)-c_{r}(n) n}{\mathrm{PoA}(I)}}=\operatorname{PoA}(I)
$$

Here, observe that the last inequality follows because $\operatorname{PoA}(I) \geq 4 / 3$, and as $C\left(x^{\mathrm{NE}}\right) \geq$ $c_{r}(n) n$. 